\title{
The PSA Controversy: To Test or Not to Test
}

\author{
Irwin S Goldstein*
}

Division of Urology, University of Pennsylvania Health System, Perelman Center for Advanced Medicine, USA

Received: January 29, 2014; Accepted: February 12, 2014; Published: February 28, 2014

*Corresponding author: Irwin S Goldstein, Division of Urology, Penn Medicine, University of Pennsylvania Health System, Perelman Center for Advanced Medicine, West Pavilion, 3rd Floor 3400 Civic Center Boulevard Philadelphia, PA 19104, USA, Tel: 215-615-0408; Fax: 215-662-3955; E-mail: Irwin. SGoldstein@uphs.upenn.edu

\section{Editorial}

The U.S. Preventive Task Force recommendation in 2012 was against PSA screening and testing for all men.

The PSA blood test was not developed to be nor was it intended to be a diagnostic test for the determination of prostate cancer. The PSA test is not cancer specific nor is it diagnostic for prostate cancer. It has been an extremely useful tool for monitoring a patient with a diagnosis of prostate cancer.

There are exceptions to this. In those patients who have an anaplastic prostate cancer with a high Gleason score, the PSA may be below the normally accepted cut off point of 4.0 and thus is of no advantage in monitoring the patient with prostate cancer, nor as a tool in diagnosing the disease.

Richard J. Ablin, PhD, DSc, a research professor of pathology at the University of Arizona who discovered and developed the PSA test has stated that this test is not cancer specific, is not diagnostic of prostate cancer, and cannot distinguish an indolent from an aggressive cancer [1].

The other potential etiologies of elevated PSA's are infections or inflammations of the prostate or of other organs of the male genital and lower urinary system, an enlarged prostate size and increased patient age.

This being accepted, the PSA blood test is however an extremely and highly useful and utilitarian tool for which to direct the urologist to the early detection of prostate cancer.

Although Dr. Ablin and others are actively pursuing new tests, both genetic and biomarker type tests, which would possess a more increased specificity and selectivity for detection of prostate cancer and for determination of indolence or aggressiveness of the cancer in the particular individual, the state of the art at this juncture in time is the PSA and PSA testing for screening and potential determination, diagnosis and treatment of this disease.

Instead of making every effort to regress in medical advancement and march backward in time, as the proponents of dissolution of the use of the PSA test- specifically the United States Preventative Services Task Force would have us do - we need to utilize the advancements we currently have at our disposal while we wait for the further advancements to develop.

It is very curious to me that, although the members of this task force are of high caliber and reputable, this recommendation was proposed by a task force which included members of the specialties of pediatrics, gynecology as well as primary specialties, epidemiology, as well as nurse specialist, etc., but the task force was devoid of members of any specialty who have direct, in-depth and comprehensive understanding, expertise and appreciation of prostate cancer and the PSA test such as urologists, medical oncologist and radiation oncologists.

It is also curious to me that as a governmental agency, quite knowledgeable regarding the governmental outlay of payment for this test for both those obtaining this by way of the Veterans Administration Health System and by Medicare and Medicaid and with the cost saving to the government of well over 3 billion dollars annually by the elimination of this life saving and life altering blood test, it has forcefully recommended that PSA testing is of no advantage and should not be performed.

It is irrefutable that there are great differences regarding the pre-PSA era compared to the post-PSA era.

1. We are now able to detect and identify prostate cancer in its curable stage $70 \%-80 \%$ of the time.

2. In the pre-PSA era we were able to detect and identify prostate cancer in its curable stage in less than $20 \%$ of the time.

3. In the pre-PSA era we identified advanced disease from prostate cancer in greater than $80 \%$ of the patients.

4. Now we see less than $20 \%$ of patients on initial evaluation with advanced and metastatic prostate cancer.

This has all been substantiated by several studies and analyses of the data.

"The European Randomized Trial of Screening for Prostate Cancer (ERSPC) reported a relative risk reduction in prostate cancer mortality of $21 \%$ " [1].

Dr. William Catalona of Northwestern University Medical Center has pointed out also that this USPTF had "disregarded epidemiologic data on prostate cancer stage migration and 
mortality" from the post PSA era as compared to the pre PSA era.

Lowering the rate of advanced prostate cancer at diagnosis reduces suffering and death from prostate cancer; patients with advanced disease have more treatments and complications; and in all screening trials, there was a lower rate of advanced disease in the screening arm [2].

In a study at the University of Rochester Medical Center, "without the use of PSA screening the number of men presenting with cases of metastatic prostate cancer would be three times greater than the actual number observed" $[2,3]$.

A 2012 European study confirmed a reduction in death rates from prostate cancer in men screened for the disease [4]. In the United States since the PSA era in the 1990's, mortality from prostate cancer has decreased from 42,000 deaths per year to 28,000 deaths per year [4].

Regarding complications due to diagnostic and therapeutic procedures for prostate cancer, it is recognized that there exists complications in less than $5 \%$ of prostate biopsies performed, and that there exists voiding and erectile issues of $20-60 \%$ secondary to radiation and surgical treatments of this disease, however the combined and overall benefit/risk ratio precludes the disregard for the benefit of the use of the PSA test for screening and early detection of the prostate cancer.

It would be a travesty of science, a travesty of the medical profession, a travesty to humanity and a step tantamount to medical malpractice to deny PSA testing to the American and world community.

\section{References}

1. Roxanne Nelson (2012) PSA testing continues to polarize medical community. Medscape.

2. Catalona WJ (2012) The argument to continue prostate cancer screening, early detection. Urology times.

3. Scosyrev E, Wu G, Mohile S, Messing EM (2012) Prostate-specific antigen screening for prostate cancer and the risk of overt metastatic disease at presentation: analysis of trends over time. Cancer 118(23): 5768-5776.

4. Ian Gram (2012) No PSA screening would triple metastatic prostate cancer cases at diagnosis. Physicians Practice. 